\title{
A Preconditioned Multisplitting and Schwarz Method for Linear Complementarity Problem
}

\author{
Cuiyu Liu and Chen-liang Li \\ School of Mathematics and Computing Science, Guilin University of Electronic Technology, Guilin, Guangxi 541004, China \\ Correspondence should be addressed to Chen-liang Li; chenliang_li@hotmail.com
}

Received 21 November 2013; Accepted 6 June 2014; Published 23 June 2014

Academic Editor: Changbum Chun

Copyright ( 2014 C. Liu and C.-1. Li. This is an open access article distributed under the Creative Commons Attribution License, which permits unrestricted use, distribution, and reproduction in any medium, provided the original work is properly cited.

The preconditioner presented by Hadjidimos et al. (2003) can improve on the convergence rate of the classical iterative methods to solve linear systems. In this paper, we extend this preconditioner to solve linear complementarity problems whose coefficient matrix is $M$-matrix or $H$-matrix and present a multisplitting and Schwarz method. The convergence theorems are given. The numerical experiments show that the methods are efficient.

\section{Introduction}

Many science and engineering problems are usually induced as linear complementarity problems (LCP): find an $x \in R^{n}$ such that

$$
x \geq 0, \quad A x-f \geq 0, \quad x^{\top}(A x-f)=0,
$$

where $A=\left[a_{i j}\right] \in R^{n \times n}$ is a given matrix and $f \in R^{n}$ is a vector. It is necessary to establish an efficient algorithm to solve the complementarity problem. Numerical methods for complementarity problems fall in two major kinds, direct and iterative methods. There have been lots of works on the solution of the linear complementarity problem ([1-4], etc.), which presented feasible and essential techniques for LCP. Recently some parallel multisplitting iterative methods for solving the large sparse linear complementarity problems are presented ([5-11], etc.). These methods are based on several splittings of the system matrix $A$ and are constructed with a suitable weighting combination of the solution of the sublinear complementarity problems.

For the large sparse linear complementarity problem, some accelerated modulus-based matrix splitting iteration methods and modulus-based synchronous two-stage multisplitting iteration methods are constructed $[7,11]$. Numerical results show that these methods are more efficient.

Many researchers have studied preconditioners applied to linear system

$$
A x=b,
$$

so that the corresponding iterative methods, such as Jacobi or GS, converge faster than the classical ones. Hadjidimos et al. [12] considered the following preconditioner:

$$
\begin{aligned}
& P_{1}(\alpha) \equiv I+S_{1}(\alpha) \\
& =\left(\begin{array}{cccccc}
1 & & & & \\
-\alpha_{2} a_{21} & 1 & & & & \\
\vdots & \ddots & & & \\
-\alpha_{i} a_{i 1} & & 1 & & \\
\vdots & & & \ddots & \\
-\alpha_{n} a_{n 1} & & & & 1
\end{array}\right) \text {, }
\end{aligned}
$$

where $\alpha=\left[0, \alpha_{2}, \ldots, \alpha_{i}, \ldots, \alpha_{n}\right] \in R^{n}$ with constants $\alpha_{i} \geq 0$, $i=2(1) n$. 
Consider

$$
S_{1}(\alpha)=\left(\begin{array}{cccccc}
0 & & & & \\
-\alpha_{2} a_{21} & 0 & & & & \\
\vdots & & \ddots & & & \\
-\alpha_{i} a_{i 1} & & & 0 & & \\
\vdots & & & & \ddots & \\
-\alpha_{n} a_{n 1} & & & & & 0
\end{array}\right)
$$

In (3), let $\alpha_{i}=1, i=2(1) n ; P_{1}(\alpha)$ is a preconditioner presented by Milaszewicz [13]. It eliminates the elements of the first column of $A$ below the diagonal. Reference [12] shows that the new modifications and improvements of the original preconditioners can improve on the convergence rates of the classical iterative methods (Jacobi, GS, etc.).

In this paper, with multisplitting technique, we will extend the preconditioner to solve the linear complementarity problem (1) and present a new multisplitting and Schwarz method. The new method is parallel and has high computational efficiency.

In Section 2, some preliminaries for the new method are presented. A multisplitting and Schwarz method is given in Section 3. Convergence analysis is given in Section 4. Section 5 presents the numerical experiments results.

\section{Preliminaries}

At first we briefly describe the notations. In $R^{n}$ and $R^{n \times n}$, the relation $\geq$ denotes the natural components partial ordering. In addition, for $x, y \in R^{n}$, we write $x>y$ if $x_{i}>y_{i}, i=$ $1,2, \ldots, n$. A nonsingular matrix $A=\left(a_{i j}\right) \in R^{n \times n}$ is termed $M$-matrix, if $a_{i j} \leq 0$ for $i \neq j$ and $A^{-1} \geq 0$. Or the nonsingular matrix $A=\left(a_{i j}\right) \in R^{n \times n}$ is called $M$-matrix, if $A=s I-C$, $C \geq 0$, and $\rho(C)<s$. Its comparison matrix $\langle A\rangle=\left(\alpha_{i j}\right)$ is defined by $\alpha_{i i}=\left|a_{i i}\right|$ and $\alpha_{i j}=-\left|a_{i i}\right|(i \neq j)$. $A$ is said to be an $H$-matrix if $\langle A\rangle$ is an $M$-matrix. To simplify the notation, we may assume that $a_{i i}=1, i=1(1) n$.

Lemma 1 (see [2]). Let $A$ be an $M$-matrix and let $x$ be a solution of (1).

(1) If $f_{i}>0$, then $x_{i}>0$ and therefore $\sum_{j=1}^{n} a_{i j} x_{j}-f_{i}=0$.

(2) If $f \leq 0$, then $x=0$ is the solution of (1).

If the problem (1) has a nonzero solution, there at least exists an index $k, f_{k}>0$. In this paper, let us assume that $f_{1}>0$. By Lemma 1 , we have the following conclusion.

Lemma 2 (see [14]). Let $A$ be an $M$-matrix, $\widetilde{A}(\alpha)=P_{1}(\alpha) A \equiv$ $\left[\widetilde{a}_{i j}\right]$, and $\widetilde{f}(\alpha)=P_{1}(\alpha) f \equiv \widetilde{f}$. If $f_{1}>0$, then the following linear complementarity problem

$$
x \geq 0, \quad \widetilde{A}(\alpha) x-\tilde{f}(\alpha) \geq 0, \quad x^{\top}(\widetilde{A}(\alpha) x-\tilde{f}(\alpha))=0
$$

is equivalent to the problem (1).
Lemma 3 (see $[15]$ ). Let $A=\left[a_{i j}\right] \in R^{n \times n}$ and $a_{i j} \leq 0$ for $i \neq j$. $A$ is an M-matrix if and only if there exists a positive vector $y$ such that $A y>0$.

Definition 4 (see [16]). (1) A splitting $A=M-N$ is termed a regular splitting of matrix $A$ if $M^{-1} \geq 0$ and $N \geq 0$.

(2) A splitting $A=M-N$ is termed $M$-splitting of matrix $A$ if $M$ is an $M$-matrix and $N \geq 0$.

(3) $A$ splitting $A=M-N$ is termed $H$-compatible splitting of matrix $A$ if $\langle A\rangle=\langle M\rangle-|N|$.

Lemma 5 (see [16]). Let $A=M_{1}-N_{1}=M_{2}-N_{2}$ be two regular splittings of $A$, where $A^{-1} \geq 0$.

(1) If $N_{2} \geq N_{1} \geq 0$, then

$$
0 \leq \rho\left(M_{1}^{-1} N_{1}\right) \leq \rho\left(M_{2}^{-1} N_{2}\right)<1 .
$$

(2) If $M_{1}^{-1} \geq M_{2}^{-1}$, then

$$
0 \leq \rho\left(M_{1}^{-1} N_{1}\right) \leq \rho\left(M_{2}^{-1} N_{2}\right)<1 .
$$

By Lemma 5, we have the following lemma.

Lemma 6. Let $A=M-N=D-B$ be two $M$-splittings of $A$, and

$$
D=\operatorname{diag}\left\{a_{11}, a_{22}, \ldots, a_{n n}\right\} .
$$

If $M \leq D$, then $\rho\left(M^{-1} N\right) \leq \rho\left(D^{-1} B\right)<1$.

Lemma 7 (see [14]). If $A$ is an $M$-matrix, then $\widetilde{A}(\alpha)$ is a $Z$ matrix and $\widetilde{A}(\alpha)$ is also an M-matrix.

Lemma 8 (see [15]). A is a nonsingular $M$-matrix if and only if all the principal minors of $A$ are positive.

By (4), we have

$$
S_{1}(\alpha) U=\left(\begin{array}{ccccc}
0 & 0 & 0 & \cdots & 0 \\
0 & \alpha_{2} a_{21} a_{12} & \alpha_{2} a_{21} a_{13} & \cdots & \alpha_{2} a_{21} a_{1 n} \\
0 & \alpha_{3} a_{31} a_{12} & \alpha_{3} a_{31} a_{13} & \cdots & \alpha_{3} a_{31} a_{1 n} \\
\vdots & \vdots & \vdots & \ddots & \vdots \\
0 & \alpha_{n} a_{n 1} a_{12} & \alpha_{n} a_{n 1} a_{13} & \cdots & \alpha_{n} a_{n 1} a_{1 n}
\end{array}\right) .
$$

Define the following matrices:

$$
\begin{gathered}
D_{\alpha}=\operatorname{diag}\left(0, \alpha_{2} a_{21} a_{12}, \alpha_{3} a_{31} a_{13}, \ldots, \alpha_{n} a_{n 1} a_{1 n}\right), \\
L_{\alpha}=\left(\begin{array}{ccccc}
0 & 0 & \cdots & 0 & 0 \\
0 & 0 & \cdots & 0 & 0 \\
0 & \alpha_{3} a_{31} a_{12} & \cdots & 0 & 0 \\
\vdots & \vdots & \ddots & \vdots & \vdots \\
0 & \alpha_{n} a_{n 1} a_{12} & \cdots & \alpha_{n} a_{n 1} a_{1, n-1} & 0
\end{array}\right), \\
U_{\alpha}=\left(\begin{array}{ccccc}
0 & 0 & 0 & \cdots & 0 \\
0 & 0 & \alpha_{2} a_{21} a_{13} & \cdots & \alpha_{2} a_{21} a_{1 n} \\
\vdots & \vdots & \vdots & \ddots & \vdots \\
0 & 0 & 0 & \cdots & \alpha_{n-1} a_{n-1,1} a_{1 n} \\
0 & 0 & 0 & \cdots & 0
\end{array}\right) .
\end{gathered}
$$


Consider the following splittings [12]:

$$
\widetilde{A}(\alpha)=\left\{\begin{array}{l}
M_{1}(\alpha)-N_{1}(\alpha) \\
\quad=\left(I+S_{1}(\alpha)\right)-\left(I+S_{1}(\alpha)\right)(L+U) \\
M_{2}(\alpha)-N_{2}(\alpha) \\
\quad=I-\left(L+L_{\alpha}-S_{1}(\alpha)+U+U_{\alpha}+D_{\alpha}\right) \\
M_{3}(\alpha)-N_{3}(\alpha) \\
\quad=\left(I-D_{\alpha}\right)-\left(L+L_{\alpha}-S_{1}(\alpha)+U+U_{\alpha}\right) \\
M_{4}(\alpha)-N_{4}(\alpha) \\
=\left(I-\left(L-S_{1}(\alpha)\right)\right)-\left(D_{\alpha}+L_{\alpha}+U+U_{\alpha}\right) \\
M_{5}(\alpha)-N_{5}(\alpha) \\
\quad=\left(I-\left(L-S_{1}(\alpha)\right)-L_{\alpha}\right)-\left(D_{\alpha}+U+U_{\alpha}\right) \\
M_{6}(\alpha)-N_{6}(\alpha) \\
\quad=\left(I-\left(L-S_{1}(\alpha)\right)-D_{\alpha}-L_{\alpha}\right)-\left(U+U_{\alpha}\right)
\end{array}\right.
$$

Define the following matrices with the above splittings:

(i) $B \equiv M_{1}^{-1}(\alpha) N_{1}(\alpha)=L+U$;

(ii) $B^{\prime} \equiv M_{2}^{-1}(\alpha) N_{2}(\alpha)=L+L_{\alpha}+U+U_{\alpha}+D_{\alpha}-S_{1}(\alpha)$;

(iii) $B^{\prime \prime} \equiv M_{3}^{-1}(\alpha) N_{3}(\alpha)=\left(I-D_{\alpha}\right)^{-1}\left(L+L_{\alpha}+U+U_{\alpha}-\right.$ $\left.S_{1}(\alpha)\right)$

(iv) $H \equiv(I-L)^{-1} U$;

(v) $H^{\prime} \equiv M_{5}^{-1}(\alpha) N_{5}(\alpha)=\left(I-\left(L-S_{1}(\alpha)\right)-L_{\alpha}\right)^{-1}\left(D_{\alpha}+\right.$ $\left.U+U_{\alpha}\right)$

(vi) $H^{\prime \prime} \equiv M_{6}^{-1}(\alpha) N_{6}(\alpha)=\left(I-\left(L-S_{1}(\alpha)\right)-D_{\alpha}-L_{\alpha}\right)^{-1}(U+$ $\left.U_{\alpha}\right)$.

Theorem 9 (see [12]). Under the notation so far, if $A$ is an $M$ matrix, then, for any $\alpha_{i} \in[0,1](i=1,2, \ldots, n)$, there exists $y \in R^{n}, y \geq 0$, such that

$$
\begin{gathered}
B^{\prime} y \leq B y, \\
\rho\left(B^{\prime \prime}\right) \leq \rho\left(B^{\prime}\right)<1, \\
\rho\left(H^{\prime \prime}\right) \leq \rho\left(H^{\prime}\right) \leq \rho(H)<1, \\
\rho\left(H^{\prime \prime}\right) \leq \rho\left(B^{\prime \prime}\right), \quad \rho\left(H^{\prime}\right) \leq \rho\left(B^{\prime}\right), \\
\rho(H) \leq \rho(B)<1 .
\end{gathered}
$$

\section{Synchronous Multisplitting and Schwarz Method}

By Theorem 9, $\rho\left(H^{\prime \prime}\right) \leq \rho(H) \leq \rho(B)<1$. It means that the Gauss-Seidel iterative methods associated with the new preconditional matrix $\widetilde{A}(\alpha)=P_{1}(\alpha) A$ will be no worse than the ones corresponding to $A$. Similar to [6], we present a synchronous multisplitting and Schwarz algorithm corresponding to $\widetilde{A}(\alpha)$.
Algorithm 10 (synchronous multisplitting and Schwarz method). (1) Give an initial vector $x^{0}, k=0$.

(2) Let

$$
x^{k+1}=\sum_{i=1}^{m} E_{i} y^{k, i},
$$

where $\sum_{i=1}^{m} E_{i}=I, E_{i}$ is a nonnegative diagonal matrix, and $y^{k, i}$ is the solution of the following LCP:

$$
\begin{gathered}
y^{k, i} \geq 0, \\
M_{i} y^{k, i} \geq F^{k}, \\
\left(y^{k, i}\right)^{\top}\left(M_{i} y^{k, i}-F^{k}\right)=0,
\end{gathered}
$$

where $F^{k}=f+N_{i} x^{k}, \widetilde{A}(\alpha)=M_{i}-N_{i}$.

(3) Consider $k:=k+1$; if the iteration solution is convergent, stop; else, return to step (2).

Let $D=\operatorname{diag}\left\{a_{11}, a_{22}, \ldots, a_{n n}\right\}, I_{i}=\left\{j: j \in S_{i}\right\}$, and $J_{i}=S \backslash I_{i}$. Define $\widetilde{M}_{i}$ as

$$
\widetilde{M}_{i}=\left\{\begin{array}{l}
\left(\widetilde{M}_{i}\right)_{I_{i}}=A_{I_{i}}, \\
\left(\widetilde{M}_{i}\right)_{J_{i}}=D_{J_{i}}, \\
\left(\widetilde{M}_{i}\right)_{I_{i} J_{i}}=0, \\
\left(\widetilde{M}_{i}\right)_{J_{i} I_{i}}=0,
\end{array}\right.
$$

where $A_{I J}$ denotes $\left(a_{k j}\right)_{k \in I, j \in J}$ and $A_{I}$ denotes $\left(a_{k j}\right)_{k, j \in I}$.

Then the following lemma is obviously true.

Lemma 11. For each splitting $\widetilde{A}(\alpha)=\widetilde{M}_{i}-\widetilde{N}_{i}(i=1,2, \ldots, m)$, let $\widetilde{M}_{i}$ be defined by (15). Then the subproblem (14) is equivalent to the following problem: find $y^{k, i} \in R^{n}$, such that

$$
\begin{gathered}
y^{k, i} \geq 0, \\
A_{I_{i}} y_{I_{i}}^{k, i} \geq F_{I_{i}}^{k}, \\
D_{J_{i}} y_{J_{i}}^{k, i} \geq F_{J_{i}}^{k}, \\
\left(y_{I_{i}}^{k, i}\right)^{\top}\left(A_{I_{i}} y_{I_{i}}^{k, i}-F_{I_{i}}^{k}\right)=0, \\
\left(y_{J_{i}}^{k, i}\right)^{\top}\left(D_{J_{i}} y_{J_{i}}^{k, i}-F_{J_{i}}^{k}\right)=0 .
\end{gathered}
$$

\section{Convergence Analysis}

In this section, we give the convergence analysis of the algorithm.

Lemma 12 (see [6]). Let $x^{*}$ be the solution of (1), and $y^{k, i}$ is the solution of (14); then

$$
\left|y^{k, i}-x^{*}\right| \leq M_{i}^{-1} N_{i}\left|x^{*}-x^{k}\right| .
$$


Theorem 13. Let $A$ be an M-matrix; the sequence $\left\{x^{k}\right\}$ generated by Algorithm 10 converges to the solution of (1).

Proof. The conclusion easily resulted from Lemma 2, Lemma 12, and Theorem 9.

In Lemma 7, if $i \neq 1,0 \leq \alpha_{i} \leq 1$, then $\widetilde{A}(\alpha)$ is an $M$ matrix. If $i \neq 1, \alpha_{i} \geq 1$, then $\widetilde{a}_{i j}=\left(1-\alpha_{i}\right) a_{i 1} \geq 0$ and $\widetilde{A}(\alpha)$ is not an $M$-matrix. In the sequel we will examine that $\widetilde{A}(\alpha)$ is an $H$-matrix with positive diagonal elements, where $\alpha_{i}(i \neq 1)$ satisfies some conditions.

Lemma 14 (see [16]). Let $A$ be either a strictly diagonally dominant or an irreducibly dominant matrix. Then $A$ is an $\mathrm{H}$ matrix.

Lemma 15. Let $A$ be a diagonally dominant $M$-matrix. If $i \neq 1$ and for $a_{i 1} \neq 0,1 \leq \alpha_{i} \leq\left(\sum_{j=1}^{n} a_{i j}-2 a_{i 1}\right) /\left(-a_{i 1}\left(2-\sum_{j=1}^{n} a_{1 j}\right)\right)$, then $\widetilde{A}(\alpha)$ is an H-matrix with positive diagonal elements.

Proof. Note that $a_{i i}=1,0 \leq-a_{i j} \leq 1$, and $\sum_{j=1}^{n} a_{i j} \geq 0$. We have

$$
\begin{aligned}
& \frac{1}{a_{i 1} a_{1 i}}-\frac{\sum_{j=1}^{n} a_{i j}-2 a_{i 1}}{-a_{i 1}\left(2-\sum_{j=1}^{n} a_{1 j}\right)} \\
& =\frac{\left(2-\sum_{j=1}^{n} a_{1 j}\right)+a_{1 i}\left(\sum_{j=1}^{n} a_{i j}-2 a_{i 1}\right)}{a_{i 1} a_{1 i}\left(2-\sum_{j=1}^{n} a_{1 j}\right)} \\
& =\left(\left(1-a_{i 1} a_{1 i}\right)+\left(1-\sum_{j=1}^{n} a_{1 j}\right)\right. \\
& \left.+a_{1 i}\left(\sum_{j=1}^{n} a_{i j}-a_{i 1}\right)\right) \\
& \times\left(a_{i 1} a_{1 i}\left(2-\sum_{j=1}^{n} a_{1 j}\right)\right)^{-1} \\
& \geq \frac{\left(1-a_{i 1} a_{1 i}\right)+\left(-\sum_{j \neq 1} a_{1 j}\right)+a_{1 i}}{a_{i 1} a_{1 i}\left(2-\sum_{j=1}^{n} a_{1 j}\right)} \\
& =\frac{\left(1-a_{i 1} a_{1 i}\right)+\left(-\sum_{j \neq 1, i} a_{1 j}\right)}{a_{i 1} a_{1 i}\left(2-\sum_{j=1}^{n} a_{1 j}\right)} \\
& >0 \text {, } \\
& \frac{\sum_{j=1}^{n} a_{i j}-2 a_{i 1}}{-a_{i 1}\left(2-\sum_{j=1}^{n} a_{1 j}\right)} \geq \frac{-2 a_{i 1}}{-a_{i 1}\left(2-\sum_{j=1}^{n} a_{1 j}\right)} \\
& =\frac{2}{\left(2-\sum_{j=1}^{n} a_{1 j}\right)}>1 .
\end{aligned}
$$

TABLE 1: Comparison of MMS and GSOR with unpreconditioned and preconditioned method.

\begin{tabular}{lcc}
\hline & Methods & Iterative steps \\
\hline \multirow{2}{*}{ GSOR } & Unpreconditioned & 311 \\
& Preconditioned & 267 \\
\hline \multirow{2}{*}{ MMS } & Unpreconditioned & 488 \\
& Preconditioned & 397 \\
\hline
\end{tabular}

$\alpha_{i}$ is well defined. By the definition of $\widetilde{A}(\alpha)$, and for $i \neq 1,1 \leq$ $\alpha_{i} \leq\left(\sum_{j=1}^{n} a_{i j}-2 a_{i 1}\right) /\left(-a_{i 1}\left(2-\sum_{j=1}^{n} a_{1 j}\right)\right)$, we have that

(1) $\sum_{j=1}^{n} \widetilde{a}_{1 j}=\sum_{j=1}^{n} a_{1 j}>0$;

(2) $\tilde{a}_{i i}=1-\alpha_{i} a_{i 1} a_{1 i}>0$;

(3) if $i \neq 1$,

$\tilde{a}_{i i}-\sum_{j \neq i}\left|\tilde{a}_{i j}\right|$

$$
\begin{aligned}
& =1-\alpha_{i} a_{i 1} a_{1 i}-\left(1-\alpha_{i}\right) a_{i 1}+\sum_{j \neq i, 1}\left(a_{i j}-\alpha_{i} a_{i 1}\right) a_{1 j} \\
& =\left(\sum_{j=1}^{n} a_{i j}-2 a_{i 1}\right)-\alpha_{i}\left(2-\sum_{j=1}^{n} a_{1 j}\right) a_{i 1}
\end{aligned}
$$

$>0$.

It implies that $\widetilde{A}(\alpha)$ is a diagonally dominant matrix; then it is an $H$-matrix with positive diagonal elements by Lemma 14.

Since $\widetilde{A}(\alpha)$ is an $H$-matrix, according to [8], we can solve the problem (5) using Algorithm 10, where $\widetilde{A}(\alpha)=M_{i}-N_{i}$ maybe an $H$-compatible splitting of matrix $\widetilde{A}(\alpha)$.

Lemma 16 (see [6]). Let $x^{*}$ be the solution of (1), and $y^{k, i}$ is the solution of (14); then

$$
\left|y^{k, i}-x^{*}\right| \leq\left\langle M_{i}\right\rangle^{-1}\left|N_{i}\right|\left|x^{*}-x^{k}\right| .
$$

Similar to the proof in Theorem 2.1 in [8], we have the following convergence theorem.

Theorem 17. Let $A$ be an M-matrix; the sequence $\left\{x^{k}\right\}$ generated by Algorithm 10 converges to the solution of the problem (1).

\section{Numerical Experiments}

In this section, we give two numerical examples to show that the new methods are efficient. In the numerical experiments, the stop criterion is $\left\|x^{k+1}-x^{k}\right\|<10^{-8}$. In the tables, MMS denotes Algorithm 10 with preconditioner, and GSOR denotes Algorithm 10, in which $m=1$.

Example 1. We consider a linear complementarity problem, whose coefficient matrix is 
TABLE 2: Comparison of MMS and GSOR with preconditioned methods ( $M$-matrix).

\begin{tabular}{lcr}
\hline$N * N$ & $\begin{array}{c}\text { MMS } \\
\text { Iterative steps }\end{array}$ & $\begin{array}{c}\text { GSOR } \\
\text { Iterative steps }\end{array}$ \\
\hline $32 * 32$ & 18 & 30 \\
$64 * 64$ & 20 & 31 \\
128128 & 21 & 33 \\
\hline
\end{tabular}

TABLE 3: Comparison of MMS and GSOR with preconditioned methods ( $H$-matrix).

\begin{tabular}{|c|c|c|}
\hline \multirow{2}{*}{$N * N$} & MMS & GSOR \\
\hline & Iterative steps & Iterative steps \\
\hline $32 * 32$ & 21 & 30 \\
\hline $64 * 64$ & 21 & 31 \\
\hline $128 * 128$ & 22 & 33 \\
\hline
\end{tabular}

TABLE 4: Comparison of MMS and AMAOR.

\begin{tabular}{|c|c|c|c|}
\hline \multicolumn{2}{|c|}{$N * N$} & MMS & AMAOR \\
\hline \multirow{2}{*}{$10 * 10$} & cputime & 0.51 & 0.42 \\
\hline & iter & 7 & 5 \\
\hline \multirow{2}{*}{$20 * 20$} & cputime & 5.49 & 8.53 \\
\hline & iter & 7 & 6 \\
\hline \multirow{2}{*}{$30 * 30$} & cputime & 24.88 & 52.76 \\
\hline & iter & 8 & 7 \\
\hline \multirow{2}{*}{$40 * 40$} & cputime & 79.34 & 192.68 \\
\hline & iter & 8 & 7 \\
\hline \multirow{2}{*}{$50 * 50$} & cputime & 205.94 & 589.48 \\
\hline & iter & 8 & 7 \\
\hline \multirow{2}{*}{$60 * 60$} & cputime & 447.80 & 1402.20 \\
\hline & iter & 8 & 7 \\
\hline
\end{tabular}

$$
A=\left(\begin{array}{ccccccccc}
1.0000 & -0.0301 & -0.1632 & -0.0280 & -0.1875 & -0.0189 & -0.1504 & -0.2652 & -0.1088 \\
-0.0926 & 1.0000 & -0.0382 & -0.1213 & -0.1520 & -0.1037 & -0.1835 & -0.1276 & -0.1509 \\
-0.1081 & -0.0901 & 1.0000 & -0.0965 & -0.0948 & -0.1823 & -0.0263 & -0.2096 & -0.1733 \\
-0.2045 & -0.1359 & -0.2263 & 1.0000 & -0.2379 & -0.0352 & -0.0117 & -0.0395 & -0.0929 \\
-0.2401 & -0.0800 & -0.0773 & -0.1115 & 1.0000 & -0.0511 & -0.1132 & -0.2230 & -0.0753 \\
-0.2245 & -0.2053 & -0.0534 & -0.0652 & -0.1381 & 1.0000 & -0.1080 & -0.0979 & -0.0898 \\
-0.1181 & -0.0751 & -0.0095 & -0.1791 & -0.1056 & -0.1595 & 1.0000 & -0.0879 & -0.1874 \\
-0.1773 & -0.0097 & -0.1900 & -0.1973 & -0.0891 & -0.0420 & -0.1320 & 1.0000 & -0.1504 \\
-0.1180 & -0.1129 & -0.1054 & -0.1694 & -0.0715 & -0.1706 & -0.0727 & -0.1085 & 1.0000
\end{array}\right),
$$

The results are shown as Table 1.

Example 2. Let us consider the following problem:

$$
x \geq 0, \quad A x-q \geq 0, \quad x^{\top}(A x-q)=0,
$$

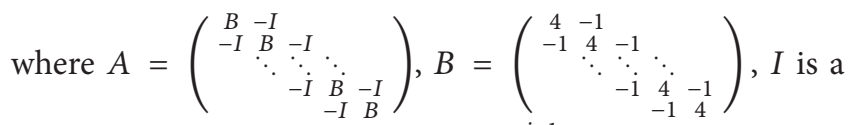
unit matrix, $q=\left(q_{i}\right)_{i=1}^{n}$, and $q_{i}=(-1)^{i+1}$.
For $a_{i 1} \neq 0$, let us choose $\alpha_{i}=0.5$; then $A(\alpha)$ is an $H$ matrix. In Algorithm $10 \widetilde{A}(\alpha)=M_{i}-N_{i}$ maybe an $H$-compatible splitting for each splitting. The corresponding results are shown in Tables 2 and 3.

An accelerated modulus-based accelerated overrelaxation (AMAOR) iteration method is presented by Zheng and Yin [11]. Same as in [11], we choose $\alpha=1.2, \mu=4$, and $\gamma=2$. In Example 2, $A=A+\mu I$. In Table 4, iter denotes iterative step and cputime denotes time (seconds). Table 4 shows that 
our preconditioned method MMS spends less time than the AMAOR.

\section{Conflict of Interests}

The authors declare that there is no conflict of interests regarding the publication of this paper.

\section{Acknowledgments}

This work was supported by the National Nature Science Projects of China 11161014 and Guangxi Experiment Center of Information Science Foundation.

\section{References}

[1] Z.-Z. Bai, "The convergence of parallel iteration algorithms for linear complementarity problems," Computers and Mathematics with Applications, vol. 32, no. 9, pp. 1-17, 1996.

[2] R.-W. Cottle, J.-S. Pang, and R. E. Stone, The Linear Complementarity Problem, Academic Press, San Diego, Calif, USA, 1992.

[3] D.-H. Li, J.-P. Zeng, and Z. Zhang, "Gaussian pivoting method for solving linear complementarity problem," Applied Mathematics - A Journal of Chinese Universities, vol. 12, no. 4, pp. 419-426, 1997.

[4] O. L. Mangasarian, "Solution of symmetric linear complementarity problems by iterative methods," Journal of Optimization Theory and Applications, vol. 22, no. 4, pp. 465-485, 1977.

[5] Z.-Z. Bai and D. Evans, "Matrix multisplitting relaxation methods for linear complementarity problems," International Journal of Computer Mathematics, vol. 63, no. 3-4, pp. 309-326, 1997.

[6] Z.-Z. Bai and D. J. Evans, "Matrix multisplitting methods with applications to linear complementarity problems: parallel asynchronous methods," International Journal of Computer Mathematics, vol. 79, no. 2, pp. 205-232, 2002.

[7] Z.-Z. Bai and L.-L. Zhang, "Modulus-based synchronous twostage multisplitting iteration methods for linear complementarity problems," Numerical Algorithms, vol. 62, no. 1, pp. 59-77, 2013.

[8] C.-L. Li and J.-P. Zeng, "Multisplitting iteration schemes for solving a class of nonlinear complementarity problems," Acta Mathematicae Applicatae Sinica, English Series, vol. 23, no. 1, pp. 79-90, 2007.

[9] Z.-Q. Luo and P. Tseng, "On the convergence of a matrix splitting algorithm for the symmetric monotone linear complementarity problem," SIAM Journal on Control and Optimization, vol. 29, no. 5, pp. 1037-1060, 1991.

[10] N. Machida, M. Fukushima, and T. Ibaraki, "A multisplitting method for symmetric linear complementarity problems," Journal of Computational and Applied Mathematics, vol. 62, no. 2, pp. 217-227, 1995.

[11] N. Zheng and J.-F. Yin, "Accelerated modulus-based matrix splitting iteration methods for linear complementarity problem," Numerical Algorithms, vol. 64, no. 2, pp. 245-262, 2013.

[12] A. Hadjidimos, D. Noutsos, and M. Tzoumas, "More on modifications and improvements of classical iterative schemes for M-matrices," Linear Algebra and Its Applications, vol. 364, pp. 253-279, 2003.
[13] J. P. Milaszewicz, "Improving Jacobi and Gauss-Seidel iterations," Linear Algebra and Its Applications, vol. 93, pp. 161-170, 1987.

[14] C. Liu and C. Li, "A new preconditioned generalised AOR method for the linear complementarity problem based on a generalised Hadjidimos preconditioner," East Asian Journal on Applied Mathematics, vol. 2, pp. 94-107, 2012.

[15] E.-L. Yip, "A necessary and sufficient condition for $M$-matrices and its relation to block LU factorization," Linear Algebra and Its Applications, vol. 235, pp. 261-274, 1996.

[16] A. Berman and R.-J. Plemmons, Nonnegative Matrices in the Mathematical Sciences, Academic Press, New York, NY, USA, 1979. 


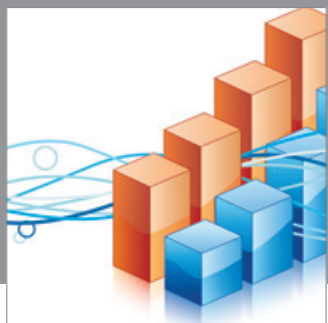

Advances in

Operations Research

mansans

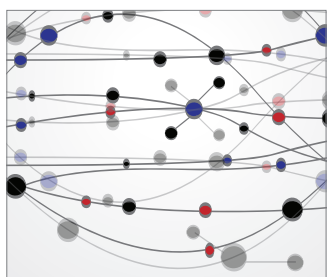

The Scientific World Journal
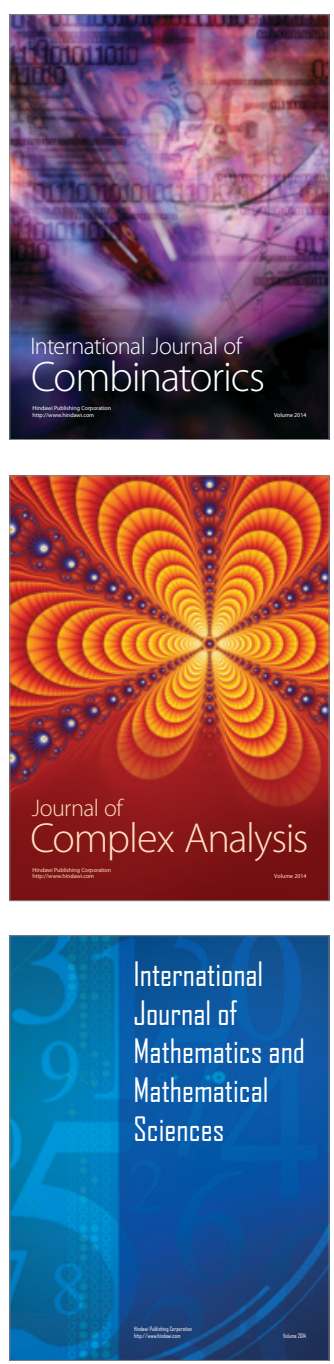
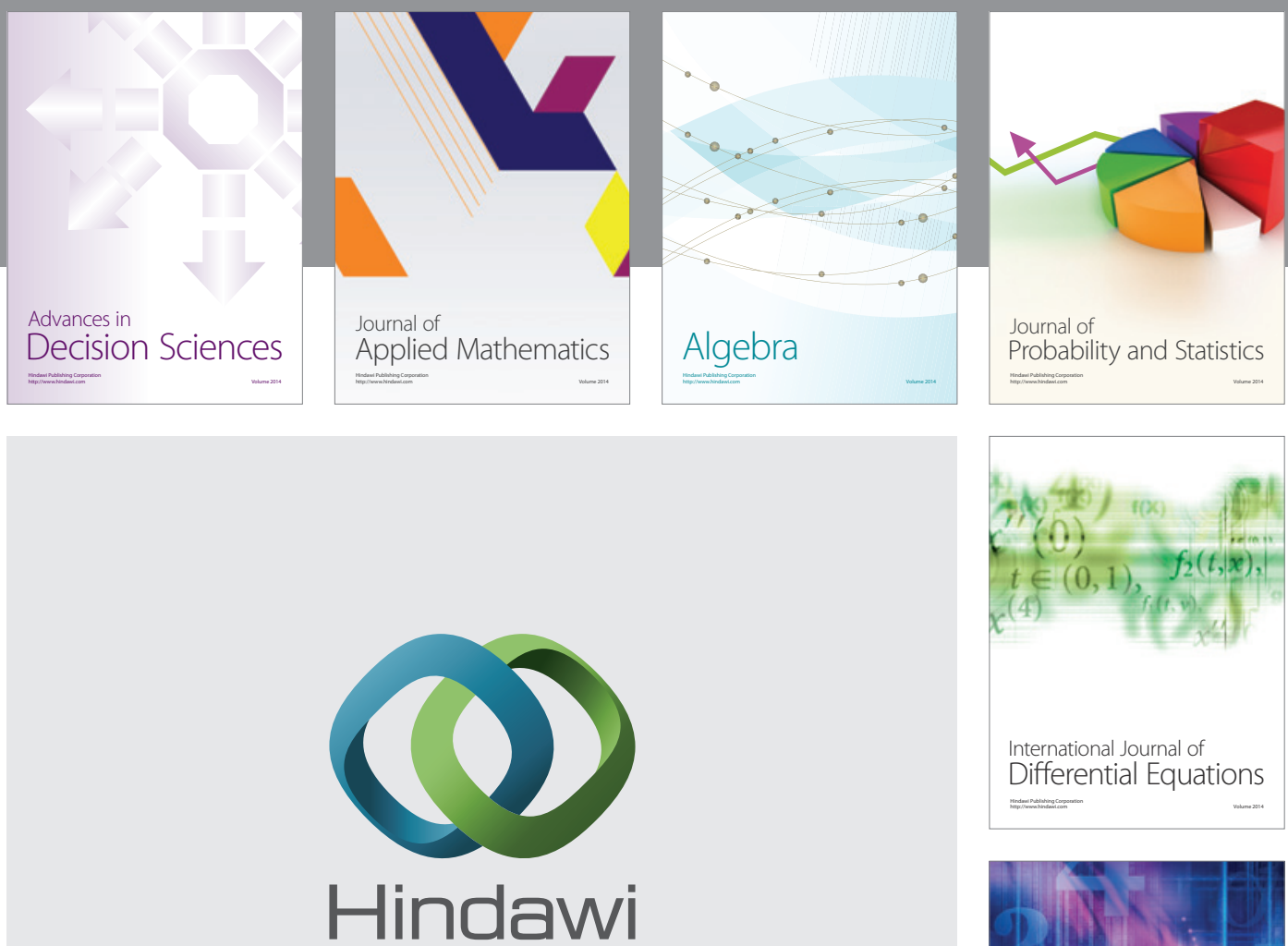

Submit your manuscripts at http://www.hindawi.com
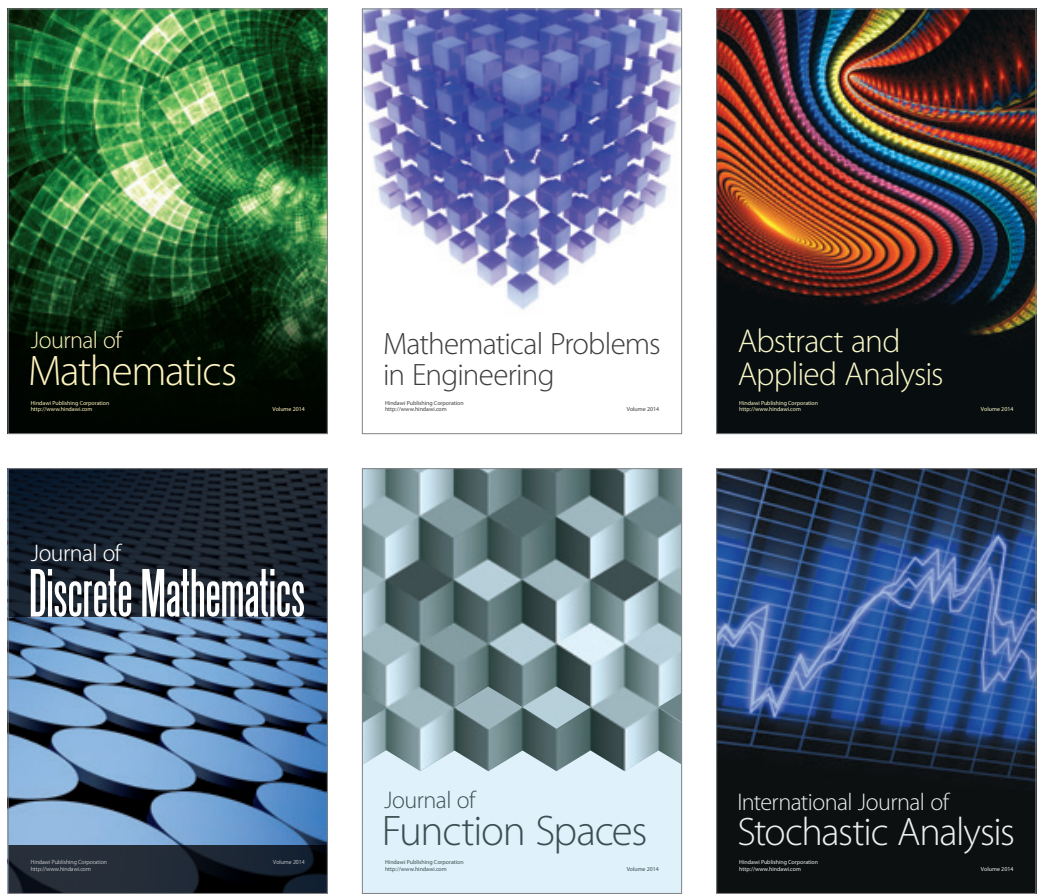

Journal of

Function Spaces

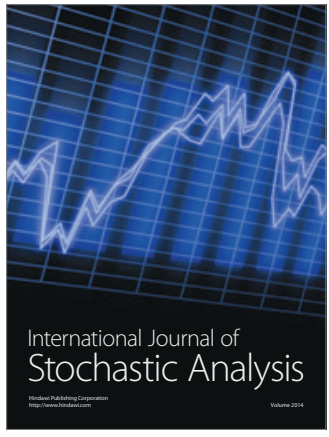

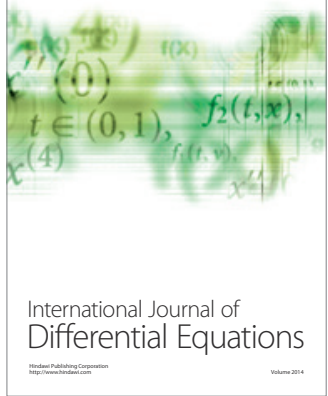
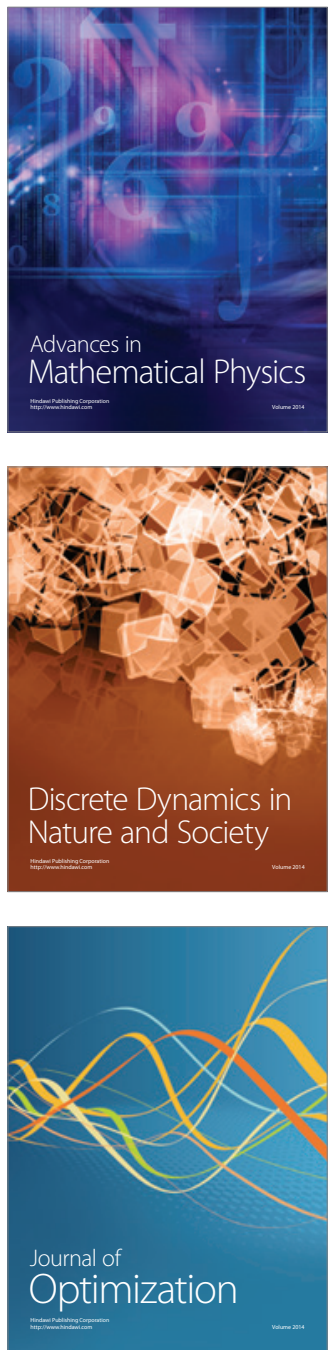